\title{
A New Approach for Acoustic Field Interpolation
}

\author{
Iu. D. Chyrka
}

Institute of Information and Communication Technologies, BAS, 1113 Sofia, Bulgaria Email:yurasyk88@ukr.net

Abstract: The present paper describes the basic performance of the 18 microphone acoustic camera manufactured by Brüel \& Kjoer for acoustic field reconstruction. The new approach for acoustic field interpolation is proposed and investigated.

Keywords: near-field acoustic holography, statistically optimized holography, equivalent source method, sound field interpolation, resolution enhancement.

\section{Introduction}

The acoustic camera is aimed to locate and characterize sound sources in a wide frequency range. Usually it uses two different types of sensors - optical camera and array of microphones (many other tools like sound level meters, sound intensity probes, accelerometers, laser vibrometers, etc. can be used as well). It fuses images, received by the sensors in a complex image by representation of different amount of sound pressure or sound intensity by different colors. This image consists of an optical photo as a background and a color map describing sound field as a foreground.

The next passage consists of some preliminary information about algorithms for sensor data processing in acoustic camera systems. The most attention is paid to near-field acoustic holography. Then a short description of the research equipment manufactured by Brüel \& Kjær is given. After that, some results obtained on the real measurements are provided and a new resolution improvement approach is proposed and investigated.

\section{Noise source identification techniques}

The acoustic camera technology is based on the so-called Noise Source Identification (NSI) or Sound Field Visualization (SFV) techniques. NSI is one of the most substantial steps in optimizing the noise emission from a wide range of 
objects including vehicles, household goods, heavy machinery and smaller separate components like engines, tires, gear-boxes, exhausts, etc., [1]. The goal of NSI is to identify the most important sub-sources on an object in terms of position, frequency content and sound power radiation. Ranking of sub-sources can be used to identify where design changes will most effectively improve the overall noise radiation. SFV techniques can be useful in the context of non-destructive evaluation, underwater imaging, machine diagnosis, and so on. No prior knowledge of the source is required, since the acoustical output of the source is measured directly, and the wave field at any other point of the source-free medium can be reconstructed, either via forward propagation towards the far field or back propagation towards the very boundary of the source.

The NSI techniques generally can be divided onto two types. The first one is a spatial filtering of signals from a sensor array in order to perform signal reception from desired directions. It is implemented through beamforming, which provides a good quality images for objects on a middle and far distance. The sound map is evaluated by focusing the antenna onto some points in a plane, parallel to the antenna plane on a given distance. Generally, a weighted sum of individual microphone delayed signals is calculated for all defined points (so called Delay and Sum beamforming). The time delay for each channel depends on the positions of the evaluated point and the microphone under consideration and it is calculated in correspondence with the distance from the estimated point to the microphone. The angular resolution depends mostly on the antenna size and sound wave frequency. The directivity pattern can be characterized by one or several main beams (or lobes) with maximum signal amplification and by many false side lobes with lower signal amplification. The beamforming is usually implemented in combination with irregular antenna arrays, which generally outperform regular arrays reducing aliasing effects.

The latter NSI technique is Near-field Acoustic Holography (NAH). The origins of holography lie in the field of optics, and can be traced back to Dennis Gabor, who reported the discovery in 1948 that an optical three-dimensional representation of an object could be obtained based on the interference between a reference illuminating beam and the resulting scattered wave fronts. Later the theory of optical holography was extended to acoustics during the late 60es. [2]

$\mathrm{NAH}$ is a technique that makes it possible, based on the $2 \mathrm{D}$ recording of sound waves, called a hologram, to reconstruct the entire 3D sound field that extends in principle from the source's boundary to the measurement plane [1-4]. Acoustic holography can calculate all physical quantities related to a sound field such as a pressure, particle velocity and sound intensity. A reconstruction algorithm performs processing of sound pressures measured by microphones to obtain a map of the source surface motion and the corresponding sound field with a high spatial resolution. Precision of reconstruction depends mostly on microphone spacing distance, sound frequency and distance between source surface and measurement (holography) plane. NAH operates in low frequency rage, upper boundary of which is limited by a distance between microphones. 
The key feature of NAH is that the near-field measurement makes it possible to capture the evanescent waves radiated by the sound source and therefore to enhance the spatial resolution. It should be noted, that evanescent waves have a wavelength shorter than the acoustic wavelength and decay exponentially away from the source. These waves are important for the reconstruction process, because during inverse propagation they are amplified exponentially. This is the reason why measures in holography must be recorded close enough to the source.

As opposite to beamforming, NAH is usually performed on a regular array grid that is also required for the classic method based on the Fourier transform. Nowadays some modern NAH approaches do not require location of microphones on specific positions. Hence, they allow reconstructing of sound fields by measurements from arrays with irregular geometries, which usually are optimized for beamforming tasks.

\section{Acoustic camera equipment}

The Brüel \& Kjær (Sound and Vibration Measurement A/S) acoustic camera has characteristics, described in the specification [5]. It consists of three main blocks (see Fig. 1): a microphone array with optic camera, a front-end with input modules and a laptop with processing software. Microphone array data are transferred to input modules via the cable harness WL1297-W-004 2013W21. The acquired data is transferred to the laptop via LAN cable. The optical camera data with maximum resolution $640 \times 480$ pixels is transferred to the laptop via USB cable.

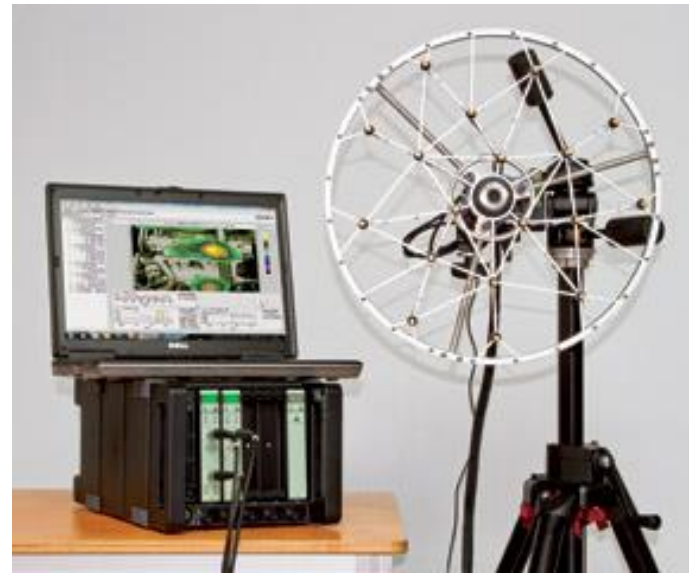

Fig. 1. The acoustic camera equipment configuration

A non-uniform slice wheel microphone array type WA-1558-W-021 with 18 microphones type 4958 is used. It covers the area with radius about $17 \mathrm{~cm}$. Microphones have sensitivity $11.2 \mathrm{mV} / \mathrm{Pa}$, operating temperature range from $10^{\circ} \mathrm{C}$ up to $55^{\circ} \mathrm{C}$ and dynamic range from $28 \mathrm{~dB}$ up to $140 \mathrm{~dB}$. Frequency range of microphones is $0.01-20 \mathrm{kHz}$. The quantization rate of the acoustic camera is $65536 \mathrm{~Hz}$ and supported upper signal frequency is $25.6 \mathrm{kHz}$. 
The front-end is the 5-slot Mainframe LAN-XI type 3660-C-000 with battery module type 2831. It is equipped with two input modules. The first one is a 6-channel input module supports Dyn-X technology, which expands its dynamic range. The second one is a 12-channel input module that does not support Dyn-X technology. Both input modules are compatible with TEDS transducers; they provide REq-X technology which flattens the result by "mirroring" the frequency responses. Data synchronization of input modules is provided by using IEEE 1588 Precision Time Protocol.

Data storage and signal processing is performed on the laptop Dell Latitude E6430 (type 7201-E-GB2) with 4-core CPU, 6 GB RAM and 1 TB HDD.

\section{NAH methods}

As an early development, Fourier NAH techniques were suggested to reconstruct sound fields of regularly shaped sources with planar, spherical, and cylindrical geometries [4]. Later many alternative methods like inverse boundary element method [6, 7], the Statistically Optimized NAH (SONAH) [8], the Helmholtz equation least squares method [9], and the Equivalent Source Method (ESM) [10, 11] were proposed. It must be noticed that these methods calculate acoustic field for the single defined frequency that must be known a priori or estimated before reconstruction, for example, by using a multichannel single frequency estimator [12]. In view of technical features of the available measurement equipment, irregular placement of microphones in particular, only SONAH (also implemented in the built-in software) and ESM are considered in this work.

The key idea in SONAH is to represent the sound pressure at desired positions on the prediction or measurement planes as the sum of $M \rightarrow \infty$ plane and evanescent waves with complex amplitudes. Therefore, the sound pressure at $N$ positions on the prediction plane can be expressed as a weighted sum of sound pressures measured at $N$ positions in the hologram plane

$$
p^{\mathrm{T}}=p_{h}^{\mathrm{T}} \mathrm{c}(r)=p_{h}^{\mathrm{T}}\left(\mathrm{A}^{\mathrm{H}} \mathrm{A}-\theta^{2} \mathrm{I}\right)^{-1} \mathrm{~A}^{\mathrm{H}} \alpha,
$$

where $p^{\mathrm{T}}$ is a transposed column vector with the $N$ pressures in the prediction plane; $p_{h}{ }^{\mathrm{T}}$ is a vector with the measured pressure; $\mathrm{c}(r)$ is the transfer matrix, which is calculated as a least square solution regularized via Tikhonov regularization; $\mathrm{A}^{\mathrm{H}} \mathrm{A}$ is a square matrix that depends on positions in the measurement (hologram) plane; $\mathrm{A}^{\mathrm{H}} \alpha$ is a square matrix that depends on the $N$ positions on the prediction plane and $N$ positions on the prediction plane; I is the identity matrix; and $\theta$ is a regularization parameter. Due to unlimited number of waves $M$ all elements of matrices $\mathrm{A}^{\mathrm{H}} \mathrm{A}$ and $\mathrm{A}^{\mathrm{H}} \alpha$ become integrals and must be evaluated numerically via Gauss and GaussLaguerre quadratures $[8,13]$.

The concept of ESM is similar to SONAH, but sound pressures at measurement plane are represented as a weighted sum of amplitudes of virtual sources arbitrary placed inside the real object or behind the real source surface. This idea actually comes from discretization of the integral of a single layer potential [7]. In the matrix form this can be written as 


$$
p_{h}=\mathrm{G}_{h v} \mathrm{q}_{v} .
$$

Here the weighting/propagation matrix $\mathrm{G}_{h v}$ consists of free-space Green's functions

$$
\mathrm{G}_{h v}(x, y)=\frac{e^{-j k r}}{4 \pi r},
$$

where $j$ denotes a complex number, $k=\omega / c$ is a wave number, $c$ is speed of sound, $\omega$ is angular frequency, and $r=|x-y|$ is a distance between measurement and evaluation points.

The unknown virtual source strengths can be calculated by inverting equation (1) as

$$
\hat{\mathrm{q}}_{v}=\left(\mathrm{G}_{h v}\right)^{+} p_{h}=\left(\mathrm{G}^{\mathrm{H}} \mathrm{G}-\theta^{2} \mathrm{I}\right)^{-1} \mathrm{G}^{\mathrm{H}} p_{h} .
$$

When these strengths are obtained, acoustical variables such as pressure or sound intensity can be reconstructed on the actual source surface. For example, sound pressure can be calculated as

$$
p_{s}=\mathrm{G}_{s v} \hat{\mathrm{q}}_{v} .
$$

The important requirement for using this method is non-zero distance between virtual sources and an actual source plane.

\section{Test results obtained by ESM \& SONAH methods}

Experimental investigation of microphone array performance was carried out in indoor conditions with presence of some reflections from variety of sound reflecting objects like room floor, ceiling, walls, office furniture and equipment. All experiments were done with the one point source of the signal. The narrowband signal central frequencies are from 0.2 up to $3 \mathrm{kHz}$. The signal was additionally prefiltered by the band-pass filter with bandwidth equals to $10 \%$ of central frequency. The input signal power is chosen large enough in comparison with ambient noise power (SNR is approximately $20 \mathrm{~dB}$ ). The acquisition time in all situations was $250 \mathrm{~ms}$, but the selected sample for processing did not exceed a few periods of the signal. Experimental results were obtained for the plane located on the actual distance to the source; the reconstruction area is limited $\pm 0.17 \mathrm{~m}$ from the center of the array in horizontal and vertical directions.

Data acquisition was done using Pulse LabShop (Customized Solution Version 17.1.2) software. There are two available options of the signal postprocessing. The first one is using of Array Acoustics Post-processing (Version 17.1.2.308) that is processing software, provided by the equipment manufacturer. Unfortunately, there were found some limitations that produce difficulties in the research. The acoustic camera field of view is approximately \pm 30 degrees in the horizontal direction and \pm 20 degrees in the vertical one. The maximum allowable distance to the object equals $24 \mathrm{~cm}$. Hence, maximum size of reconstructed sound field map is $26 \times 18 \mathrm{~cm}$, that is less than dimension of the array. 
Yet another limitation for the preinstalled post-processing is upper signal frequency around $2 \mathrm{kHz}$, which is defined by the average microphone spacing distance.

On the other hand, the developed software enables obtaining of realizations of the sound pressure map, calculated as Root-Mean-Squares (RMS) of pressure at any desired distance, signal frequency and in full size matching physical dimension of the array $(\sim 32 \times 32 \mathrm{~cm})$. It uses a matrix-form implementation of the mentioned SONAH and ESM algorithms.

Our case can be considered as a sparse array configuration, because number of microphones and array size are comparatively small. Under such conditions some resolution-enhancing reconstruction techniques must be use to obtain acceptable results. As it is shown here [1], the most preferable one is underdetermined ESM, when number of virtual sources is bigger than number of microphones. Test results have shown that number of virtual sources, which provide the best ratio between resolution and number of calculations, must be near 250 for the present array. At the same time SONAH does not have such option and directly reconstruct pressure at desired points.

Now let us consider some test cases for performance comparison of ESM and SONAH algorithms. The results of resolution comparison for different frequencies on two distances to object are joined in the Table 1. Resolution in the single point source scenario has been measured as width of the peak corresponding to target on the normalized level $-3 \mathrm{~dB}$. The results were obtained using Tikhonov regularization for both methods. Optimal regularization parameter value was calculated using Generalized Cross Validation (GCV) criterion. The retreat distance (between virtual sources and prediction plane) for ESM was chosen $0.01 \mathrm{~m}$.

Table 1. Resolution of ESM and SONAH holography methods

\begin{tabular}{|c|c|c|c|c|}
\hline \multirow{2}{*}{$\begin{array}{c}\text { Center frequency } \\
(\mathrm{Hz})\end{array}$} & \multicolumn{4}{|c|}{ Resolution $(\mathrm{cm})$} \\
\cline { 2 - 5 } & \multicolumn{2}{|c|}{ Distance $5 \mathrm{~cm}$} & \multicolumn{2}{c|}{ Distance $10 \mathrm{~cm}$} \\
\hline 200 & 12 & 14 & 12 & 14 \\
\hline 500 & 12 & 15 & 10 & 16 \\
\hline 800 & 11 & 13 & 10 & 10 \\
\hline 1100 & 10 & 13 & 9 & 12 \\
\hline 1400 & 12 & 18 & 8 & 12 \\
\hline 1700 & 8 & 16 & 8 & 19 \\
\hline 2000 & 9 & 15 & 7 & 19 \\
\hline 2800 & 7 & 12 & 7 & 19 \\
\hline
\end{tabular}

One can see that algorithms show close results in the low frequency range. But at higher frequencies SONAH has some worsening of resolution that is more significant at bigger distance.

The example of a reconstructed acoustic map is shown in Fig. 2. The raw data was captured with the acoustic camera at the distance $0.05 \mathrm{~m}$ between point source (a small loudspeaker was used) and the microphone array. Source is located in the boresight position (in front of the array center). Center frequency of narrowband source was chosen $1100 \mathrm{~Hz}$. Number of evaluation points is 576. Color bar shows correspondence of normalized values $(\mathrm{dB})$ to color gradient. Acoustic map points which level is lower than $-3 \mathrm{~dB}$ are not displayed. 


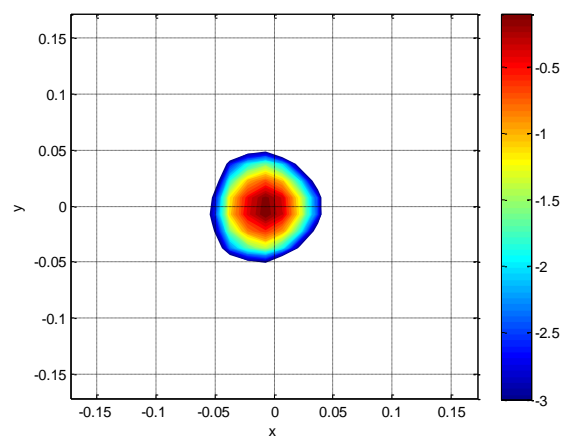

(a)

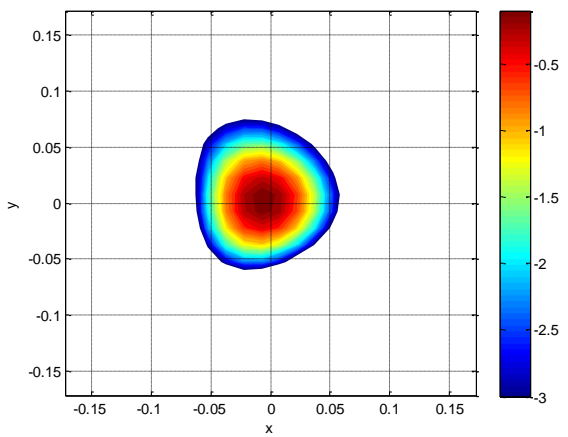

(b)

Fig. 2. Sample of reconstructed acoustic map of point sources of narrowband noise waveform with center frequency $1100 \mathrm{~Hz}$ on the distance $5 \mathrm{~cm}$ : by ESM (a); by SONAH (b)

The above-mentioned situations also were modelled by computer simulation. The considered processing algorithms have shown similar results on simulated and real data and some small differences may be observed due to indoor testing conditions.

\section{Improvement of SONAH resolution}

\subsection{Theoretical background}

When number of available measurement channel is limited, precision of reconstruction and method resolution can be improved by acoustic field interpolation and extrapolation that is also called a virtual microphone technique $[1,14,15]$. This means that measurement surface density and/or area are numerically enlarged. Let us consider the new approach to performing such interpolation that allows improvement of resolution of SONAH method in high frequency range in the task of single source localization.

The key idea is to use auto-regression relation for any sinewave signal [16]

$$
s_{i}=\alpha s_{i-1}-s_{i-2}, \quad \alpha=2 \cos (\omega \Delta t) .
$$

In other words, having signal values at any two points, time difference between them (usually it is sampling period) and signal frequency, we can calculate the signal value inside (interpolation) or outside (extrapolate) the pair of microphones. Time difference and frequency give us a phase shift (or growth) between two points. In application to acoustic field interpolation this expression defines some limitations and conditions that will be described later.

We can rewrite (2) for our case

$$
s_{2}=\alpha s_{v}-s_{1},
$$

where $s_{1}$ and $s_{2}$ are signal values from the first and second microphones. Hence, the interpolated signal from the virtual microphone has to be calculated as

$$
s_{v}=\left(s_{1}+s_{2}\right) / \alpha=\left(s_{1}+s_{2}\right) / 2 \cos (\omega \Delta t) .
$$


The angular frequency is calculated from signal frequency $F$ as $\omega=2 \pi F$. Calculation of the $\Delta t$ will be described after coordinate calculation description.

Position of the virtual microphone is calculated for each pair of microphone based on their coordinates and estimated coordinates of the sound source. Estimation of the source position is taken as argmax of a reconstructed map of sound pressure from the first stage of calculations by the conventional holography method. Let consider finding the virtual microphone coordinates on the example shown in the Fig. 3.

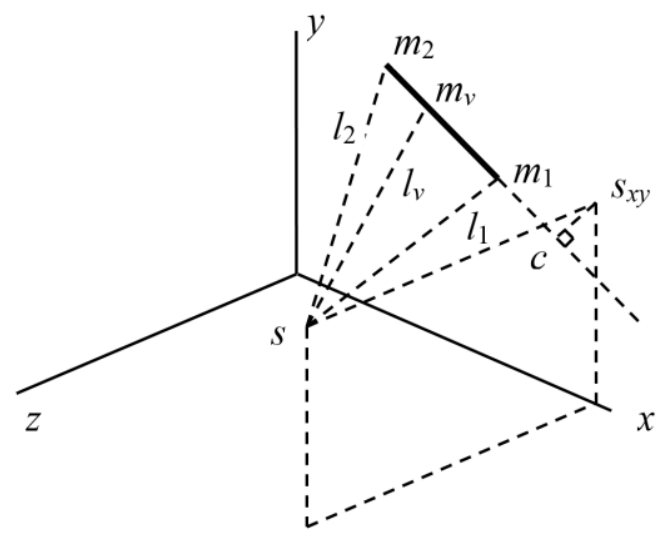

Fig. 3. Finding of the virtual microphone location

Here $m_{1}, m_{2}, m_{v}$ are positions of the real first, second and virtual microphones respectively; $s, s_{x y}$ is estimated source point and its projection on the $x y$ (holography) plane; $c$ is the point of cross-section of the line $m_{1}, m_{2}$ with a perpendicular from point $s_{x y} ; l_{1}, l_{2}, l_{v}$ define corresponding distances from the source to microphones.

It is necessary to assure the equal phase shift $\omega \Delta t$ of the interpolated signal to two other signals in the equation (3). It can be achieved if the virtual microphone is placed in the position that meets the next condition:

$$
l_{\mathrm{v}}=\left(l_{1}+l_{2}\right) / 2 \text {. }
$$

In this case coordinates $x_{v}, y_{v}$ of the virtual microphone can be calculated from the next equations:

$$
\begin{gathered}
l_{v x y}=\sqrt{l_{v}^{2}-z_{s}^{2}}, \\
d=\sqrt{\left(x_{s}-x_{c}\right)^{2}+\left(y_{s}-y_{c}\right)^{2}} \\
l_{c 1}=\sqrt{\left(x_{c}-x_{1}\right)^{2}+\left(y_{c}-y_{1}\right)^{2}}, \\
l_{c v}=\sqrt{l_{v x y}^{2}-d^{2}}, \\
x_{v}=l_{c v}\left(x_{1}-x_{c}\right) / l_{c 1}+x_{c}, \\
y_{v}=l_{c v}\left(y_{1}-y_{c}\right) / l_{c 1}+y_{c},
\end{gathered}
$$

where coordinates of the cross-section point $c$ are calculated as a solution of the equation system, which defines normality of two vectors $\overline{x_{1}, x_{2}}$ and $\overline{s_{x y}, c}$ : 


$$
\begin{gathered}
\mathrm{Ac}=\mathrm{b}, \\
\mathrm{A}=\left[\begin{array}{cc}
y_{2}-y_{1} & -\left(x_{2}-x_{1}\right) \\
-\left(x_{2}-x_{1}\right) & -\left(y_{2}-y_{1}\right)
\end{array}\right], \\
\mathrm{b}=\left[\begin{array}{c}
x_{1}\left(y_{2}-y_{1}\right)-y_{1}\left(x_{2}-x_{1}\right) \\
-x_{s}\left(x_{2}-x_{1}\right)-y_{s}\left(y_{2}-y_{1}\right)
\end{array}\right], \\
\mathrm{c}=\left[\begin{array}{l}
x_{c} \\
y_{c}
\end{array}\right]=\mathrm{A}^{\prime} \mathrm{b} .
\end{gathered}
$$

The time difference $\Delta t$ for the equation (3) can be estimated from coordinates of microphones and the sound source as

$$
\Delta t=\frac{\left|l_{1}-l_{2}\right|}{2 \lambda F},
$$

where $\lambda$ is a wave length.

Using of equation (3) requires continuous growth of phase between microphones 1-2 or vice versa, that can be achieved only if point $c$ is outside the segment $\left(m_{1}, m_{2}\right)$. This means that not every microphone pair can be used for interpolation in such a manner and positions of virtual microphones are dependent on the sound source location. The example of microphone locations for the boresight source position and distance to object $10 \mathrm{~cm}$ is shown in Fig. 4.

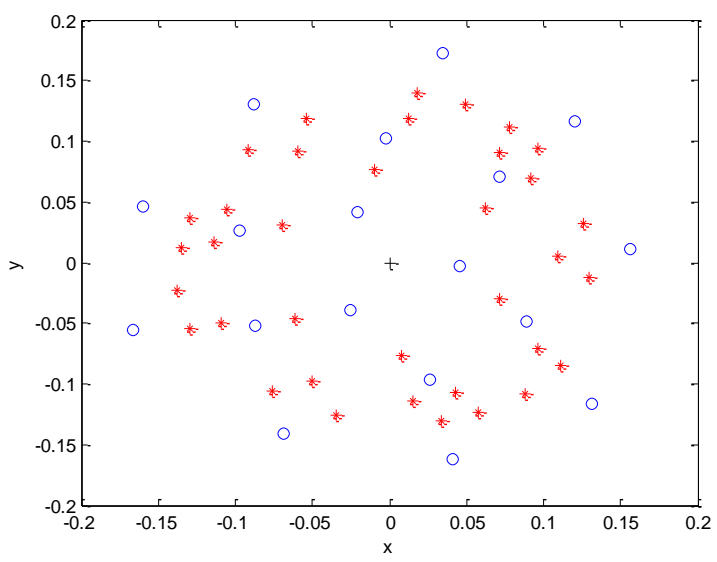

Fig. 4. Positions of virtual microphones

The black cross in the center shows the source position. Blue circles show positions of the real microphones and red asterisks represents calculated positions of virtual microphones. It is well visible that mentioned limitation on microphone placement has led to absence of microphones in the center of the array. Moreover, when source is placed too far from the center, positions of virtual microphones become highly asymmetrical and destroy the balance of energy consumption [17] that makes impossible to obtain correct acoustic field reconstruction after application of this interpolation method. 


\subsection{Experimental results}

The carried out research has shown that this interpolation technique allows increasing of SONAH resolution in frequency range upper than $2 \mathrm{kHz}$. Examples of reconstructed acoustic maps for different frequencies are shown in the Fig. 5.

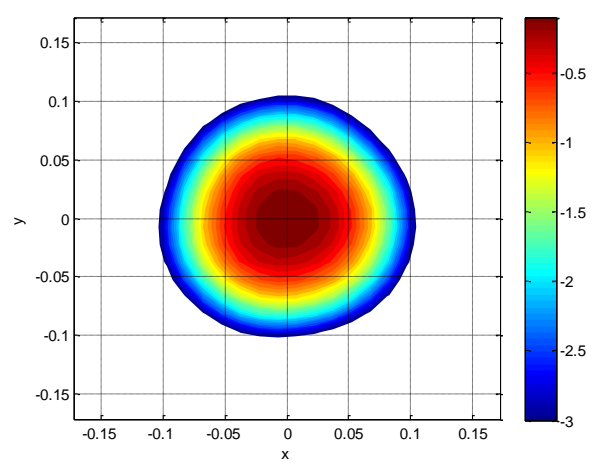

(a)

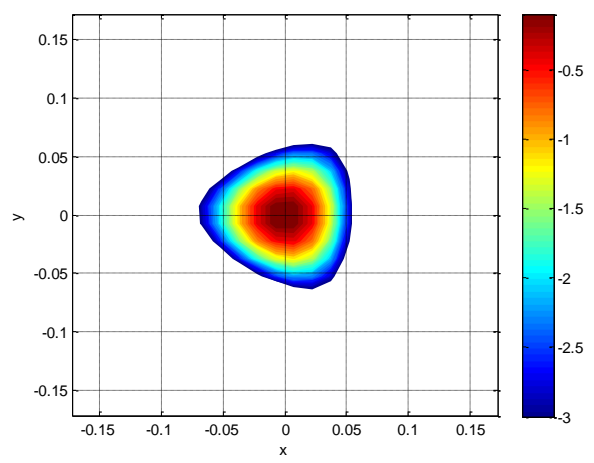

(c)

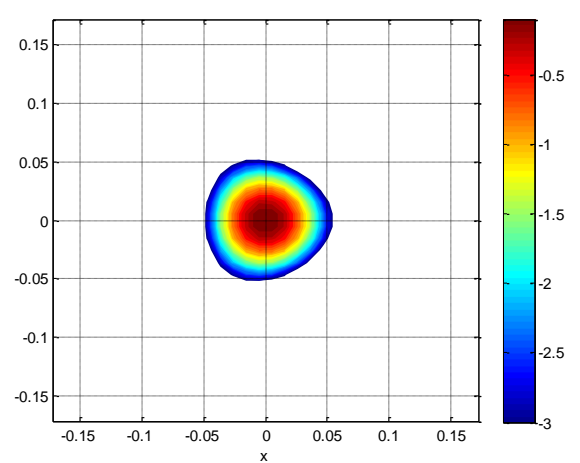

(b)

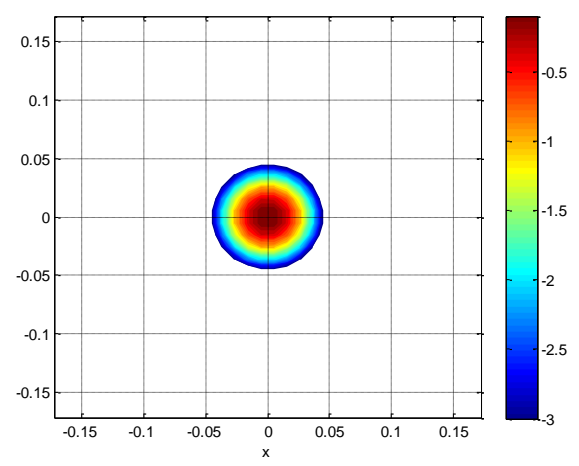

(d)

Fig. 5. Sample of reconstructed acoustic map of point sources of narrowband noise waveform with different center frequency on the distance $10 \mathrm{~cm}$ by SONAH: $2.5 \mathrm{kHz}$ without interpolation (a);

$2.5 \mathrm{kHz}$ with interpolation (b); $4 \mathrm{kHz}$ without interpolation (c); $4 \mathrm{kHz}$ with interpolation (d)

One can see, that the proposed method allows 1.5-2 times improvement in comparison to conventional one. But, at higher frequency the source must be located as close as possible to the center, because conventional method starts giving shifted peaks that has negative impact on the interpolation procedure.

Research results also have shown that this interpolation technique almost does not show any positive effect in combination with ESM. As well, research results obtained by using acoustic field extrapolation in the similar manner have not shown additional gain in results due to bigger distances between real and virtual microphones. 


\section{Conclusion}

Near-field acoustic holography is a useful technique for sound source localization A new method for resolution improvement of SONAH was proposed. It was proved that the described new method provides up to two times better resolution in the high frequency range.

Acknowledgments: The research reported in the paper was partly supported by the project AComIn "Advanced Computing for Innovation", Grant 316087, funded by the FP7 Capacity Programme (Research Potential of Convergence Regions).

\section{References}

1. B a i, R. M., J. G. I h, J. B e n e st y. Acoustic Array Systems. John Wiley \& Sons Singapore Pte. Ltd., Singapore, 2013.

2. Gra h a m, T. S. Long-Wavelength Acoustic Holography. - Journal of the Acoustical Society of America, Vol. 46, 1969, No 1A, p. 116.

3. Ma yn ard, J. D., E. G. W illi a m s, Y. Le e. Nearfield Acoustic Holography: I. Theory of Generalized Holography and the Development of NAH. - Journal of the Acoustical Society of America, Vol. 78, 1985, No 4, pp. 1395-1413.

4. W i 11 i a m s, E. G. Fourier Acoustics. Academic Press, 1999.

5. http://www.bksv.com/

6. K i m, B. K., J. G. I h. On the Reconstruction of Vibro-Acoustic Field over the Surface Enclosing an Interior Space Using the Boundary Element Method. - Journal of the Acoustical Society of America, Vol. 100, 1996, No 5, pp. 3003-3016.

7. Schuhmacher, A., J. Hald, K. B. Rasmus sen, P. C. Hansen. Sound Source Reconstruction Using Inverse Boundary Element Calculations. - Journal of the Acoustical Society of America, Vol. 113, 2003, No 1, pp. 114-126.

8. H a ld, J. Patch Near-Field Acoustical Holography Using a New Statistically Optimal Method. Brüel \& Kjær Technical Review, 2005, No 1, pp. 40-50.

9. W a n g, Z., S. F. W u. Helmholtz Equation - Least-Squares Method for Reconstruction of the Acoustic Pressure Field. - Journal of the Acoustical Society of America, Vol. 102, 1997, No 4, pp. 2020-2032.

10. S a r k i s s i a n, A. Method of Superposition Applied to Patch Near-Field Acoustic Holography. Journal of the Acoustical Society of America, Vol. 118, 2005, No 2, pp. 671-678.

11. B i, Ch., X. Chen, J. Chen, R. Zhou. Near-Field Acoustic Holography Based on the Equivalent Source Method. - Science in China Ser. E Engineering \& Materials Science, Vol. 48, 2005, No 3, pp. 338-353.

12. Ch y rka, I. D., I. P. O m e l ch u k. Multichannel Modified Covariance Estimator of a SingleTone Frequency. - Cybernetics and Information Technologies, Vol. 15, 2015, No 7, pp. 35-44.

13. J a c obse n F., V. J a u d. Statistically Optimized Near Field Acoustic Holography Using an Array of Pressure-Velocity Probes. - Journal of the Acoustical Society of America, Vol. 121, 2007, No 3, pp. 1550-1558

14. S a r k is s i a n, A. Extension of Measurement Surface in Near-Field Acoustic Holography. Journal of the Acoustical Society of America, Vol. 115, 2004, No 4, pp. 1593-1596.

15. Will i a m s, E. G. Continuation of Acoustic Near-Fields. - Journal of the Acoustical Society of America, Vol. 113, 2003, No 3, pp. 1273-1281.

16. Nikitin, A. V., S. V. I u sh a n o v. Measurement of Instantaneous Frequency of Wideband Signals on the Short Observation Interval. - Measuring Technics. 2008. No 2, pp. 50-54.

17. B in g H u a, C., Z. W e i, C. Z i N a n. Underwater Acoustic Sensor Networks Deployment Using Improved Self-Organize Map Algorithm. - Cybernetics and Information Technologies, Vol. 14, 2014, pp. 63-77 